\title{
Localized prostate cancer presenting as a renal pseudo-tumour
}

\author{
Zia Poonja, MD, FRCPC; Alan So, MD, FRCSC; Bernhard J Eigl, MD, FRCPC
}

UBC Division of Medical Oncology, British Columbia Cancer Agency, Vancouver, BC

Cite as: Can Urol Assoc J 2015;9(7-8):E505-6. http://dx.doi.org/10.5489/cuaj.2950 Published online July 17, 2015.

\section{Abstract}

Malignant obstructive uropathy can be a result of common genitourinary malignancies (i.e., ureter, prostate, bladder, or kidney) or extrinsic processes, such as colon and ovarian tumours. We present a case of uropathy caused by prostatic obstruction, which initially presented as a renal psuedo-tumour. We also review the relevant literature.

\section{Case presentation}

A 63-year-old male presented to the emergency department with a 3-month history of progressive fatigue, decreasing appetite, worsening nausea, and abdominal pain. He was in renal failure, with a creatinine of $1430 \mathrm{umol} / \mathrm{L}(16.2 \mathrm{mg} / \mathrm{dL})$. A bladder ultrasound revealed $2 \mathrm{~L}$ of urine, which responded favourably to catheterization. The patient was placed on antibiotics empirically due to his elevated white cell count, elevated lactate, and urine leukocytes. Further workup of persistent abdominal pain resulted in a non-contrast computed tomography (CT) scan of his abdomen and pelvis.

The imaging revealed a $7.3 \times 6.9 \times 5.8-\mathrm{cm}$ necrotic appearing mass in the right renal pelvis (Fig. 1a). Extension of the mass into the right kidney was reported. The bladder was circumferentially thickened. Multiple periaortic and upper abdominal lymph nodes were identified (Fig. 1b). The scan was interpreted as being in keeping with an extensive malignancy involving both kidneys, ureters and bladder likely originating in the right renal pelvis. The case likely revealed urothelial or renal cell carcincoma.

Urine cytology showed only atypical urothelial cells. A cystoscopy and retrograde pyelogram showed a hydronephrotic left ureter with no obvious filling defects, likely secondary to long-standing bladder obstruction. On the right, there was a 10-cm filling defect from the mid-proximal right ureter up to the kidney. A biopsy of the ureteric mass was attempted, but due to poor visualization from hematuria tissue, sampling was not obtained. However, prostate core biopsies were performed on the basis of a digital rectal examination showing a large and rigid feeling prostate and a presenting prostate-specific antigen of $110 \mathrm{ng} / \mathrm{mL}$. Pathology results confirmed the diagnosis of Gleason $5+4=9$ adenocarcinoma in all biopsy cores (100\% of each core).

The patient was eventually transferred to a tertiary cancer centre for further oncologic investigations and treatment, initially with a presumptive diagnosis of metastatic (node positive) urothelial carcinoma of the right collecting system, and coincidental prostate cancer. Luteinizing hormonereleasing hormone therapy was initiated, and a ${ }^{99} \mathrm{Tc}$ bone scan was negative for metastatic disease.

On review at his first medical oncology appointment, his overall condition and performance status had improved significantly. Repeat imaging was undertaken to locate a biopsy site for a tissue diagnosis. The repeat CT of his chest, abdomen and pelvis demonstrated significant improvement of the bilateral hydronephrosis with resolution of the urothelial mass previously noted. The previously seen retroperitoneal lymphadenopathy had decreased. A repeat cystoscopy was undertaken and this did not add any significant findings. There was no filling defect identified this time with a normal distal ureter and renal contour on retrograde pyelogram of the right ureteric orifice. Formal renoscopy was also normal. Careful bilateral ureterscopy demonstrated normal ureters. Urine cytology was repeated and was normal. Given the resolution the mass and normal cystoscopy, it was concluded that the previously identified pseudo-tumour was due to inflammation from urosepsis and obstruction at the level of the prostate. The patient underwent further management for high-risk prostate cancer, with plans for external beam radiation to his prostate following a transurethral resection of the prostate. 


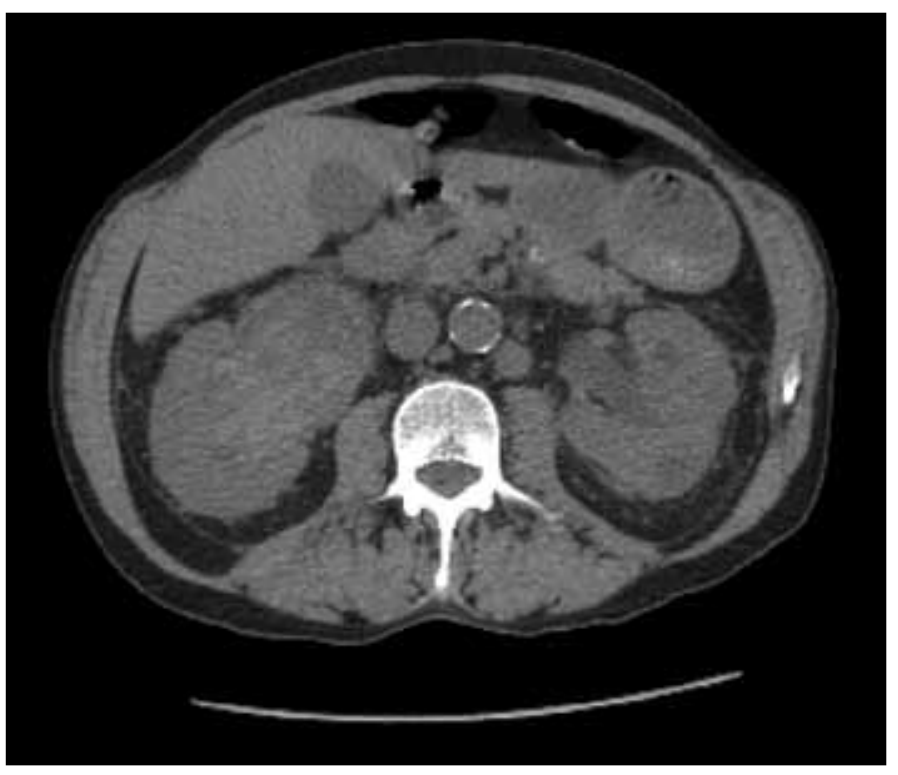

Fig. 1a. Large mass in the right renal pelvis at initial presentation.

\section{Methods}

A literature review was undertaken using PubMed, with the search terms "prostate cancer and renal tumour." Of the 419 case reports identified, none were similar to ours. To our knowledge therefore this is the first reported instance of prostate cancer presenting with imaging findings of a renal pseudo-tumour.

\section{Discussion}

This case of an unusual presentation of localized prostate cancer underlines the importance of maintaining a broad differential diagnosis and pursuing definitive tissue confirmation, especially in light of the significantly worse prognosis this man's presumptive diagnosis carried.

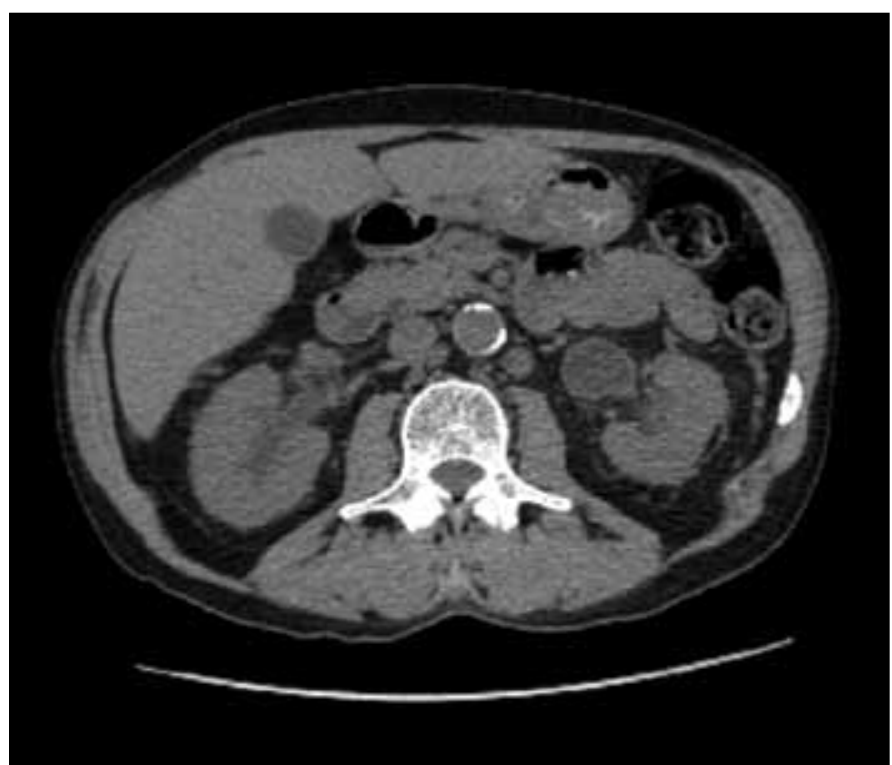

Fig. $\mathbf{1 b}$. Resolution of the mass in the right renal pelvis

\section{Conclusion}

When evaluating patients with imaging findings suggestive of malignancy without a tissue diagnosis, it is important to consider re-imaging if their performance status dramatically improves.

Competing interests: The authors all declare no competing financial or personal interests.

This paper has been peer-reviewed.

Correspondence: Dr. Zia Poonja, UBC Division of Medical Oncology, British Columbia Cancer Agency, 600 W. 10th Ave, Vancouver BC, V5Z 4E6; zia.poonja@bccancer.bc.ca 